\title{
Research on the Method for Estimating the Motion state of the Opponent's Agent in the Confrontation Environment
}

\author{
Yun Zhang ${ }^{*}$, Yang Chen $^{2}$ \\ ${ }^{1}$ School of Computer Science \& Technology, Xi'an University of Science \& Technology, Xi'an 710054 \\ Shaanxi P.R.China. \\ P.R.China. \\ * Corresponding author. Tel.: 008613571980258; email: yunzhang710@163.com \\ Manuscript submitted November 30, 2017; accepted January 10, 2018. \\ 10.17706/jsw.13.1.66-71
}

2 ThoughtWorks Software Technologies (Xi'an) Ltd, Room 06, 6th floor, Building E Huanpu Science and Technology Industrial Park No.211 8th Tiangu Road, Hi-tech Development District Xi'an, 710077, Shanxi

\begin{abstract}
In this paper, we mainly study the method of estimating the opponent's behavior in the confrontation environment. Firstly, the method of estimating the motion state of the other agent is studied. Then, the state estimation algorithm is designed, and weighted average filter is used to improve the state estimation of moving targets. Finally, the effectiveness of the algorithm is proved by experiments. Emphasizes the importance of intelligent learning.
\end{abstract}

Key words: Multi-agent, confrontation, motion state, estimating.

\section{Introduction}

In the process of intelligent confrontation, if the exact state and trend of the two sides are obtained in time, the agent can make a reasonable decision.The motion shape and the tendency of the agent can be obtained by external sensors. The state of motion between our agents is achieved by synchronous message transmission, and the motion state information of the target is obtained by motion estimation technique. In this paper, we mainly study the method of estimating the opponent's behavior in the confrontation environment [1], [2].

\section{Algorithm Research}

Suppose that the sensor transmission time is tk. The last moment sensor action is tk-1. At the moment, the position of the target is $\mathrm{Xk}$, and the position of the last moment is $\mathrm{Xk}-1$. In principle, the current velocity $\mathrm{Vk}$ of the target can be approximated by the following formula :

$$
V_{k} \approx\left|X_{k}-X_{k-1}\right| /\left(t_{k}-t_{k-1}\right)
$$

The time difference between the front and back images of the sensor is usually very small, so the formula (1) can be used to estimate the current velocity of the body.

The main factors that influence the motion estimation of the visual object are [3]: 
1) under the state of confrontation, our agents and opponents are in constant motion;

2) we need to obtain the motion parameters of the target relative to the global absolute coordinate system;

the target imaging is biased, and the resolution of the transmitted image is limited. For example, usually, even if our agent and rival target in a stationary state, the target image obtained at different times will be different, and this difference corresponding to the physical location of the target's estimated difference can be large (tens of meters to hundreds of meters).

According to the formula (1), it is necessary to obtain the change of the position of the target in the coordinate system in order to estimate the behavior of the target object.

The absolute coordinates of the target are calculated as follows:

The first step is to calculate the coordinates of the object in the local coordinate system;

The second step: use the filtering method to deal with this coordinate value;

The third step: convert the self positioning information into absolute coordinates.

The practice shows that the odometer information is accurate and reliable in a short time interval. Therefore, we use the following methods to estimate the absolute coordinates of the target system:

The absolute position coordinates of the target on the first moment image I1 are X1, which is calculated by the numerical value of the actual self positioning L1. Adjacent to another moment image is I2, which corresponds to the absolute coordinates of the target position value is $\mathrm{X} 2$, this value is obtained by the L2 conversion, L2 is calculated from the number of intelligent self positioning. The calculation method of L2 is shown in formula (2).

$$
L_{2}=L_{1}+\Delta o
$$

In the formula (2), a is the odometer value obtained by the conversion, which is in the absolute coordinate and in the interval between the two frames. The initial motion estimation procedure for visual objects is as follows:

The first step is to obtain the self localization data L1, which is generated by the current frame sensor image, and calculate the absolute coordinate position of the target object X1;

The second step: check the self positioning information, and adjust it, so that the self positioning data as accurately as possible within the allowable error range;

The third step: query Tk, the value of the current frame image time. The time difference of the previous frame image is calculated:

$$
\Delta T=T_{k}-T_{k-1}
$$

The fourth step: according to the distance information of the odometer, calculate the change $\Delta O_{\text {of }}$ the position of the agent in the time period $\Delta T$;

The fifth step: Based on the self localization data of the current frame image, calculate the self localization data $\left(L_{2}=L_{1}+\Delta o\right)$ of the agent in the adjacent frame image;

The sixth step: when the adjacent frame image appears, the agent can calculate the difference of the data from the self positioning data domain, so as to calculate the absolute coordinates X2 of the target when the adjacent frame image appears;

The seventh step: check the absolute coordinate position X2 of the target object, so that the self positioning data tend to be accurate within the allowable error range; 
The eighth step: the initial motion estimation of the target is obtained by calculating the formula (3).

$$
V_{k}=\left(X_{1}-X_{2}\right) / \Delta T
$$

Since the initial estimation value of the visual object contains random noise, it is necessary to improve the performance of motion estimation.

The filter is mainly based on two aspects:

The time series of motion estimation of the opponent's target object obtained by the agent itself;

The current estimated value of the "mate".

The process of object motion estimation:

The first step is to deal with the two adjacent frames and obtain the initial object motion estimation;

The second step: filtering noise;

The third step: smoothing filter (with the target object motion estimation method).

Research on Weighted Filtering

As a matter of fact, the target object is always kept the same behavior state in a short period of time due to the inertia and the granularity of the decision making. In other words, the state change of target behavior is limited. Therefore, it is possible to reduce the error range of motion estimation by using the weighted average of the recent motion estimation [4], [5].

We assume that the visual image is obtained using the formula (4). The estimated value of the initial motion of an object is Vk. The estimated value of the initial motion of the object is Vk-1, N, Vk-2,... , Vk-N. At the present time, the estimated value of the motion state of the target is approximately as follows:

$$
\overline{V_{k}}=\sum_{i=0}^{N} w_{i} V_{k-i}
$$

Among them, $w_{i}$ is the weight, $\sum_{i=0}^{N} w_{i}=1$.

Assuming that at the current moment, the actual state of a target object is $V_{k}^{\prime}, V_{k-1}^{\prime}, \ldots, V_{k-N}^{\prime}$. The deviation between the estimated value and the actual value is $\Delta V_{k}, \Delta V_{k-1}, \Delta V_{k-N}$. These deviations have the same probability distribution, and their mathematical expectation is 0 . By the formula (5) can be obtained for the deviation of the estimated value of motion:

$$
\Delta V_{k}^{\prime}=\overline{V_{K}}-V_{k}^{\prime}=\sum_{i=0}^{N} w_{i}\left(\Delta V_{k-i}+V_{k-i}^{\prime}-V_{k}^{\prime}\right)
$$

In the case of $V_{k}^{\prime} \approx V_{k-1}^{\prime} \approx \ldots \approx V_{k-N}^{\prime}$, the formula can be simplified as:

$$
\Delta V_{k}^{\prime} \approx \sum_{i=0}^{N} w_{i} \Delta V_{k-i}
$$

From the theory of probability and statistics, the solution of the $\Delta V_{k}^{\prime}$ in the formula is equivalent to the mathematical expectation of solving $\Delta V_{k-i}$, that is, $\Delta V_{k}^{\prime}$ tends to be close to the mathematical 
expectation of $\Delta V_{k-i}$, which is 0 .

However, in reality, the goal will often change their behavior, such as sudden acceleration or deceleration. But in a short period of time, agents tend to maintain a behavior. So this assumption is effective only in a short period of time. Therefore, we can not choose too large weighted average number $\mathrm{N}$.

Multi agent weighted filtering can reduce the deviation of motion estimation of visual objects (from the perspective of probability and statistics). Assuming that the estimated value of the behavior of other agents received by an agent is $V_{1}, V_{2}, \ldots, V_{N}$, the agent can use the weighted average method (formula 7) to further adjust the movement behavior of the target object estimation $\mathrm{V}$.

$$
V=\sum_{i=1}^{N} w_{i} V_{i} \quad \sum_{i=1}^{N} w_{i}=1
$$

From the point of view of probability and statistics theory, formally, when $w_{i}=\frac{1}{N}$, the mathematical expectation of random variables in the upper form is the same. Therefore, it is considered that formula (7) is effective in improving the behavior of target objects.

\section{Experimental and Analysis}

Suppose we have two agents, A1 and A2, who travel at the same height $(12 \mathrm{~km})$ and speed $(300 \mathrm{~m} / \mathrm{s})$. The opposing party has a stationary target on the ground. By using the above algorithm, we can estimate the moving speed of the target by our two agents.

The experimental results are shown in figure 1. Among them, the horizontal and vertical coordinates are expressed by the speed of $\mathrm{X}$ and $\mathrm{Y}$. The horizontal and vertical coordinates of the figure (a) and figure (b) are $\mathrm{m} / \mathrm{s}$.

From the experimental results of Figure 1 we can see that our two agents to estimate the opponent's static state of the target, the state estimate is approximately $V=0(\mathrm{~m} / \mathrm{s})$, that is the actual value of the object.However, it can be seen that the estimated error is relatively large. This requires a more accurate method to estimate and predict. You can imagine that if all agents in our opponents together to estimate the target, and on the basis of the "learning", to strengthen the multi-agent experience, then estimate and forecast accuracy will be greatly improved.

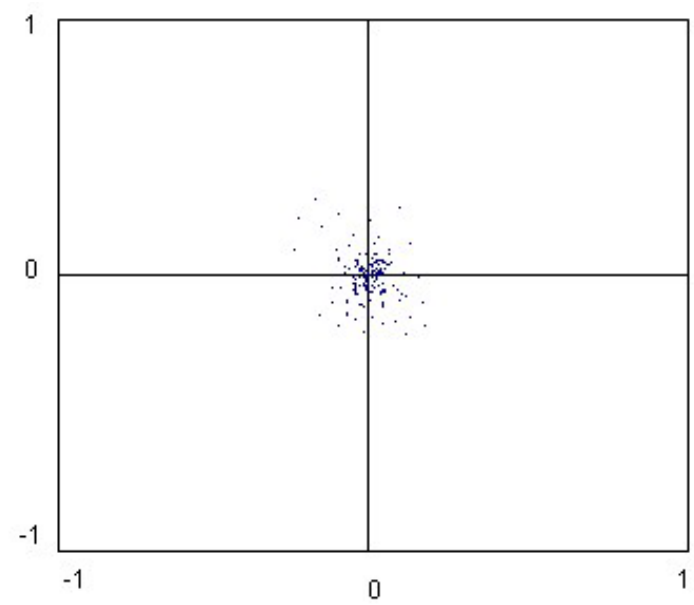

(a). The estimated value of the intelligent agent A1(for the opponent's target state) 


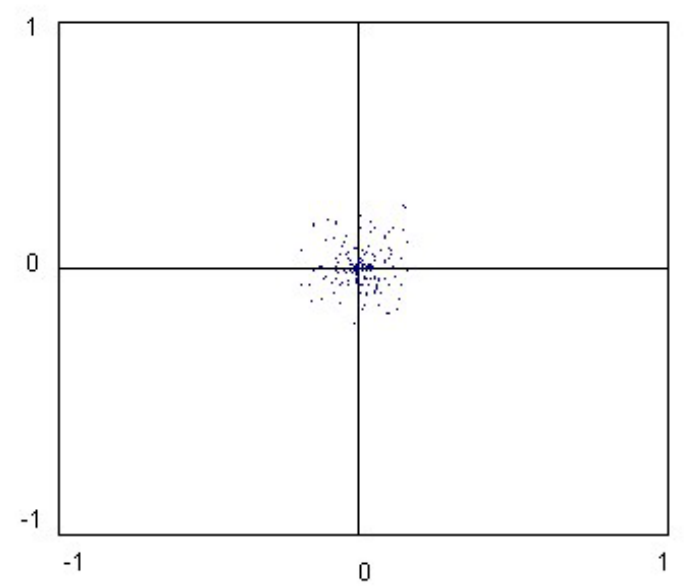

(b)The estimated value of the intelligent agent A2(for the opponent's target state)

Fig. 1. Estimation of target motion by our two agents.

\section{Conclusion}

One of the most important tasks of the confrontation is to seek a way of making decisions in the case of the current confrontation environment, and thus get an effective strategy to cope with the current situation.

In this paper, the method of estimating the motion state of the other agent is studied. The state estimation algorithm is designed, and weighted average filter is used to improve the state estimation of moving targets. Finally, the effectiveness of the algorithm is proved by experiments. Emphasizes the importance of intelligent learning.

\section{Acknowledgment}

This research was financially supported by the foundation of special science research project of Shaanxi Provincial Education Bureau in 2017 (17JK0513) and the cultivation fund of scientific research of Xi'an University of Science and Technology (201634).

\section{References}

[1] Zhang, Y., Li, W. H., \& Chen, Y. (2011). Applying ACMC strategy to multidimensional data mining. Journal of Northwestern Polytechnical University, 29(3), 418-423.

[2] Yun, Z., \& Li, W. Hua. (2012). AHP construct mining component strategy applied for data mining process. Proceedings of the 2012 IEEE International Conference on Information Science and Technology Wuhan.

[3] Niazi, M., \& Hussain, A. (2011). Agent-based computing from multi-agent systems to agent-based models: A visual survey. Scientometrics, 89(2), 479-499.

[4] Tzafestas, S. G., Tzamtzi, M. P., \& Rigatos, G. G. (2002). Robust motion planning and control of mobile robots for collision avoidance in terrains with moving objects. Mathematics and Computers in Simulation (MATCOM).

[5] Ghasemlou, S., Ali, M., Taher, A. S., \& Mohammadreza, T. (2014). Homecoming: A multi-robot exploration method for conjunct environments with a systematic return procedure. European Conference on Multi-Agent Systems, Springer International Publishing, 111-127. 


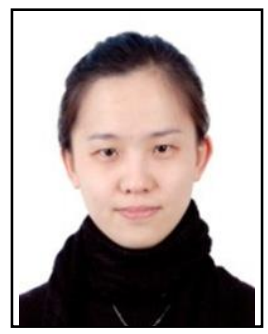

Zhang Yun was born in Xi'an, Shaanxi, China, in 1983. She graduated from School of computer science and technology, Northwestern Polytechnical University (Xi'an Shaanxi, China), obtained a PhD in computer science and technology in 2014, and the research field is intelligent decision support system .

She worked as a teacher at Xi'an University of Science And Technology after graduation. Work address: School of Computer Science \& Technology, Xi'an University of Science \& Technology, Xi'an 710054 Shaanxi P.R.China, and the research field is intelligent decision support system . 